\title{
Validation of the Simplified UVB Model to Assess the Pharmacodynamics of Analgesics in Healthy Human Volunteers
}

\author{
Kuntheavy Ing Lorenziniab ${ }^{\text {, Marie Besson }}{ }^{\text {ab }}$, Youssef Daaliab, Denis Salomon ${ }^{\text {bc }}$, Pierre Dayer ${ }^{\text {ab }}$, \\ and Jules Desmeules ${ }^{\star a b}$
}

\begin{abstract}
A pharmacokinetic/pharmacodynamic, randomized, crossover, placebo-controlled study was conducted in healthy human volunteers with the primary objective of exploring the existence of a positive interaction between paracetamol $1 \mathrm{~g}$ and ketorolac $20 \mathrm{mg}$ intravenously on experimental pain models. Further, the simplified UVB model was validated as a screening tool for analgesics or a combination of analgesics in clinical drug development. It was observed that the UVB irradiation induced primary hyperalgesia, evidenced by significant decreases of the heat pain threshold from (mean \pm SD) $46.9 \pm 1.1^{\circ} \mathrm{C}$ to $40.1 \pm 1.7^{\circ} \mathrm{C}(p<0.001)$ and of the mechanical pain threshold (62\% decrease). A small intra- and inter-individual variability was observed as well as consistent intra-day stability for the heat pain threshold. The UVB irradiation also resulted in the development of an area of secondary hyperalgesia of $21.3 \pm 11.3 \mathrm{~cm}^{2}$. The mechanical pain threshold and area of secondary hyperalgesia showed small intra-individual variability and consistent intra-day stability. However, a greater variability was observed between subjects, which suggests that a crossover design would allow limiting the number of subjects.
\end{abstract}

Keywords: Analgesics combination · NSAIDs · Paracetamol · UVB · Variability

\section{Introduction}

Paracetamol is often combined with nonsteroidal anti-inflammatory drugs (NSAIDs) in the management of acute pain. ${ }^{[1-3]}$ The rationale underlying the practice of combining drugs for pain management is mainly based on the consideration that combining drugs that act at different receptors and on different pain mechanisms might enhance pain relief. Paracetamol acts mainly on the brain and spinal cord; nevertheless, the exact mechanism of action has not been fully elucidated. ${ }^{[4]}$ The prostaglandin $\mathrm{H}_{2}$ synthase (PGHS), ${ }^{[5]}$ the serotoninergic system, ${ }^{[6,7]}$ and/or the cannabinoid system ${ }^{[8,9]}$ have been proposed as potential targets. The action of NSAIDs

${ }^{*}$ Correspondence: Prof. J. Desmeules ${ }^{\mathrm{ab}}$

Tel.: +4122 3829942

Fax: +41223829945

E-mail: jules.desmeules@hcuge.ch

aDivision of clinical pharmacology and toxicology

University Hospitals of Geneva

Rue Gabrielle-Perret-Gentil 4, CH-1211 Geneva

${ }^{b}$ Faculty of Medicine

University of Geneva

1 rue Michel-Servet, $\mathrm{CH}-1211$ Geneva 4

'Division of Dermatology and Venerology

University Hospitals of Geneva

Rue Gabrielle-Perret-Gentil 4, CH-1211 Geneva mainly relies on the inhibition of prostaglandin biosynthesis at the site of the inflammation, ${ }^{[10]}$ although some NSAIDs also exhibit a central action. ${ }^{[11]}$

The hypothesis of our study is that the combination of paracetamol and ketorolac, a NSAID, results in an additive effect, involving both peripheral and central nociceptive pathways. This hypothesis was tested by performing a pharmacokinetic/pharmacodynamic (PK/PD) study in healthy volunteers. For this purpose, an analytical method was developed for measuring the plasma concentrations of the test drugs and thereby assessing their pharmacokinetics (PK). The pharmacodynamics (PD) was assessed using an experimental pain model that involved peripheral and central pathways. The detailed methods and the results of this analytical method ${ }^{[12]}$ and of this PK/PD clinical study ${ }^{[13]}$ have been published elsewhere.

The present article is aimed at describing from a methodological point of view the validation of the simplified UVB model, also called the 'sunburn' model, which has been used to characterize the PD of paracetamol and ketorolac in our study. Further, it is also aimed at determining whether this model could be feasibly deployed as a screening tool of analgesics or analgesics combination targeting different sites of action (peripheral and central) and mechanisms involved (primary and secondary hyperalgesia) early in clinical drug development, in healthy volunteers.

\section{Materials and Methods}

\subsection{Study Design}

A randomized, double-blind, crossover, controlled, phase 1 study was performed. The four study arms were: 1) combined intravenous (iv) paracetamol 1 $\mathrm{g}$ and iv ketorolac $20 \mathrm{mg}$; 2) iv paracetamol $1 \mathrm{~g}$; 3) iv ketorolac $20 \mathrm{mg}$; and 4) placebo (sodium chloride $0.9 \%$ ). The study was approved by the local Ethics Committee and the Swiss Agency for Therapeutic Products (Swissmedic), and was conducted in accordance with Good Clinical Practice.

\subsection{Subjects}

A total of 12 healthy, pain-free, nonsmoking, skin type-III, 20 to 50 year-old male volunteers were recruited. The exclusion criteria were contraindications to paracetamol and NSAIDs and intake of other analgesics and skin diseases. Informed consent was obtained in written form after providing adequate verbal and written information.

\subsection{Pharmacodynamics Assessment}

Experimental models of pain are useful tools for characterizing the analgesic effects of drugs. ${ }^{[14,15]}$ Models inducing hyperalgesia can mimic clinical pain which is often associated with signs of hyperalgesia. The sunburn model uses UVB irradiation to induce stable primary and secondary hyperalgesia. ${ }^{[16]}$ The primary area 
of hyperalgesia is explained by sensitization of peripheral nociceptor terminals and peripheral pain hypersensitivity, while the secondary hyperalgesia involves a central mechanism of sensitization. ${ }^{[17]}$ The detailed methodology used in this study is described elsewhere. ${ }^{[13]}$ The sunburn model is used in a simplified way as previously described ${ }^{[18]}$ for assessing the central and peripheral analgesic effect of paracetamol and ketorolac, respectively. The sunburn model has been characterized and validated by another research group; ${ }^{[16]}$ however it has not been fully characterized when used in a simplified way, ${ }^{[18]}$ that is by estimating the dose of UVB according to the volunteers' skin type. Moreover this model has never been used for assessing the effect of a combination of analgesics that act on different targets. The volunteers were exposed to twice the minimal erythema doses (MED) of UVB light (UV801KL, Waldmann, Villingen-Schwenningen, Germany) on a $3 \times 3 \mathrm{~cm}$ area on the glabrous part of the non-dominant forearm, $20 \mathrm{~h}$ prior to each study session, as described previously.[18] The primary hyperalgesia was assessed by measurement of the heat and mechanical pain thresholds (HPT and MPT, respectively). The HPT, defined as the lowest temperature producing pain, was assessed using a $9 \mathrm{~cm}^{2}$ Peltier thermode (Medoc Advanced Medical Systems, Ramat-Yishai, Israel), placed in contact with the erythematous skin. ${ }^{[19]}$ The MPT, defined as the lowest pressure producing pain, was assessed using an electronic von Frey device (Bioseb, Id-Tech Bioseb, Chaville, France) as described previous1y. ${ }^{[20]}$ The MPT was also measured around the erythema (secondary hyperalgesia) and in a control skin zone (contra lateral side). The secondary hyperalgesia was assessed by determination of the area of secondary hyperalgesia to pinprick, which was measured on the skin surrounding the erythema using a rigid von Frey hair $(462 \mathrm{mN})$ along eight linear paths as described previously. ${ }^{[21]}$ Subsequent to the transcription of the eight points on a paper, the area was calculated according to the weight, using standards of 9 and $25 \mathrm{~cm}^{2}$.

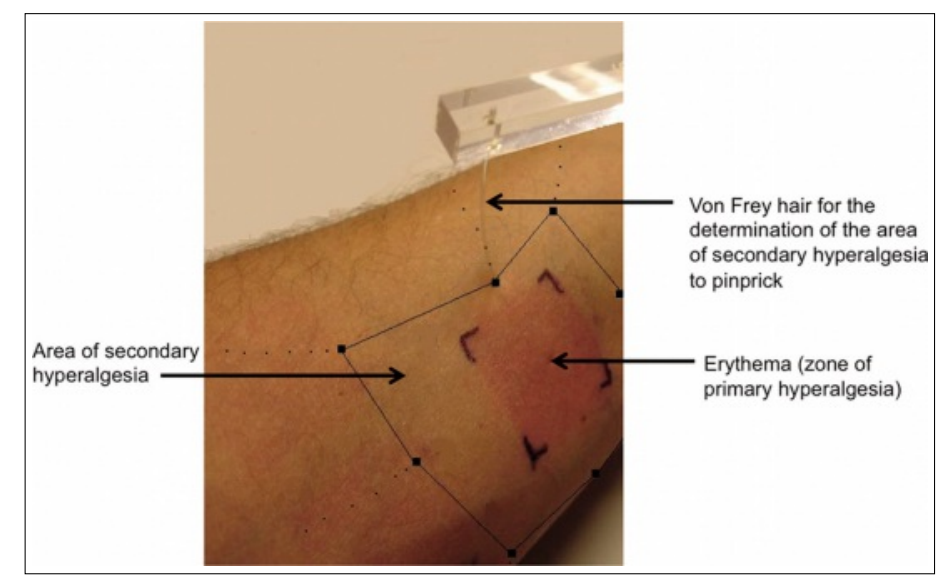

Fig. 1. Zones of primary and secondary hyperalgesia. This figure shows the determination of the area of secondary hyperalgesia to pinprick using a rigid von Frey hair along eight linear paths.

The four study sessions were conducted at least a week apart. The PD measures were performed at baseline and at 1, 2, 4, 6 and $24 \mathrm{~h}$ after drug administration. The last evaluation $(24 \mathrm{~h})$ represented $44 \mathrm{~h}$ after UVB irradiation $(20 \mathrm{~h}$ after irradiation +24 $\mathrm{h}$ after drug administration). The PD data measured in the placebo arm, as well as at baseline in the other study groups were used for characterizing the induced hyperalgesia in terms of variability.

\subsection{Statistical Analysis}

Statistical analysis was performed using SPSS 11.0 (SPSS Inc, Chicago, IL, USA). All results were expressed as the mean \pm SD. Variance components were estimated to quantify the intra- and inter-individual variability of the sunburn model.

\section{Results}

A total of 13 subjects were screened for the study. Among these, two volunteers withdrew from the study (one for unknown reasons and the other for medical reasons). Therefore, 11 volunteers completed the study; their mean age was $26.0 \pm 9.8$ years old. No serious adverse event was associated with the UVB irradiation or with the study drugs.

Table 1. Variability estimates associated with the UVB model

\begin{tabular}{|l|lllll|}
$\begin{array}{l}\text { Component of } \\
\text { variability }\end{array}$ & \multicolumn{4}{l}{ Coefficient of variation [\%] } & \\
& HPT & $\begin{array}{l}\text { MPT, } \\
\text { primary } \\
\text { hyperal- } \\
\text { gesia }\end{array}$ & $\begin{array}{l}\text { MPT, } \\
\text { secondary } \\
\text { hyperal- } \\
\text { gesia }\end{array}$ & $\begin{array}{l}\text { MPT, } \\
\text { control skin } \\
\text { zone }\end{array}$ & $\begin{array}{l}\text { Area of } \\
\text { secondary } \\
\text { hyperal- } \\
\text { gesia }\end{array}$ \\
$\begin{array}{l}\text { Within-subject } \\
\text { within-day }\end{array}$ & 1.5 & 20.8 & 13.9 & 11.9 & 14.4 \\
$\begin{array}{l}\text { Within-subject } \\
\text { between-day }\end{array}$ & 2.6 & 32.0 & 23.2 & 27.5 & 29.7 \\
Between subject & 4.3 & 49.6 & 46.1 & 45.8 & 49.8
\end{tabular}

\subsection{Pharmacodynamics Assessment}

The detailed results of this study regarding the effects of the test drugs have been published elsewhere. ${ }^{[13]}$ Here the focus is on the characteristics of the sunburn model, as applied in the research unit. It was observed that the UVB irradiation caused reproducible skin inflammation in all the subjects, which was evident by visible erythema (Fig. 1). An erythema developed in all volunteers without exception, during each study session. When compared to the pre-irradiation value, the HPT was found to significantly decrease $20 \mathrm{~h}$ after UVB irradiation, from $46.9 \pm$ $1.1^{\circ} \mathrm{C}$ to $40.1 \pm 1.7^{\circ} \mathrm{C}(p<0.001)$. A small intra- and inter-individual variability was observed for the HPT, with a coefficient of variation of $2.6 \%$ for within-subject between-day measures and of $4.3 \%$ for between subject measures (Table 1). The HPT remained remarkably stable throughout the study day (20 to $26 \mathrm{~h}$ after exposure), with a coefficient of variation of $1.5 \%$ for within-subject within-day measures. Primary hyperalgesia to heat stimuli was still present $44 \mathrm{~h}$ after UVB exposure $\left(\mathrm{HPT}=41.4 \pm 2.0^{\circ} \mathrm{C}\right)$. Fig. 2 depicts the decrease of the HPT induced by UVB irradiation within the zone of erythema and its stability throughout the study day. It could been seen that the UVB irradiation induced significant decreases in MPT, from 198.8 $\pm 76.5 \mathrm{~g}$ to $74.7 \pm 34.8 \mathrm{~g}$ in the zone of erythema, representing a decrease of $62 \pm$ $14 \%(p<0.002)$, and from $198.8 \pm 76.5 \mathrm{~g}$ to $129.9 \pm 58.8 \mathrm{~g}$ around the erythema (33 $\pm 27 \%)(p<0.04)$. For primary hyperalgesia, the observed intra- and inter-individual variability was $32.0 \%$ and $49.6 \%$, respectively (Table 1 ). For secondary hyperalgesia, the observed intra- and inter-individual variability was $23.2 \%$ and $46.1 \%$, respectively. Further, the MPT remained remarkably stable throughout the study day (20 to $26 \mathrm{~h}$ after exposure), with a coefficient of variation of $20.8 \%$ and $13.9 \%$ in the primary and secondary zones, respectively, for within-subject within-day measures. 


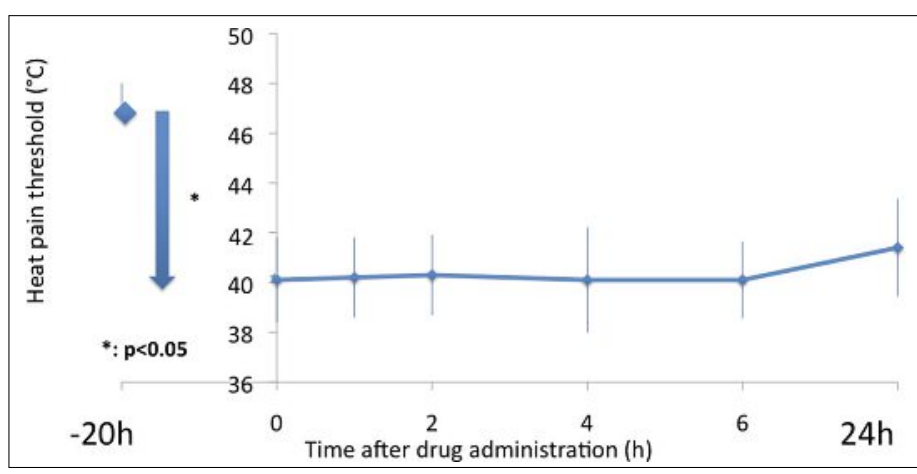

Fig. 2. Heat pain threshold before the irradiation and between 20 and $44 \mathrm{~h}$ after irradiation (0 to $24 \mathrm{~h}$ after drug administration). Data are expressed as means $\pm \mathrm{SD} ; \mathrm{N}=11$.

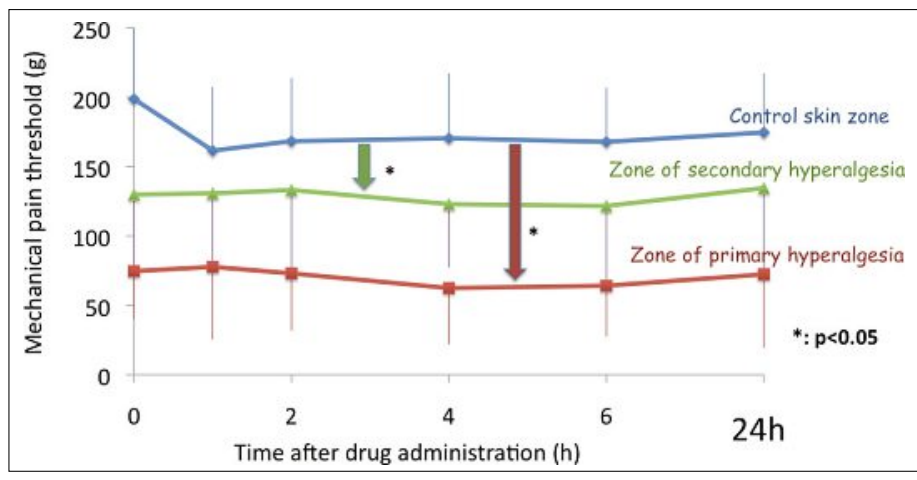

Fig. 3. Mechanica pain threshold at three different sites of measurement assessed between 20 and $44 \mathrm{~h}$ after irradiation ( 0 to $24 \mathrm{~h}$ after drug administration). Data are expressed as means $\pm \mathrm{SD}$; $\mathrm{N}=11$.

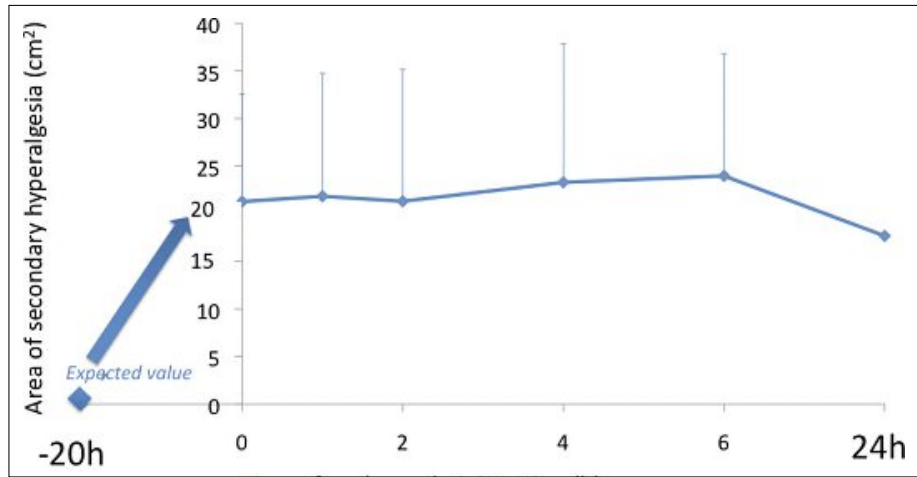

Fig. 4. Area of secondary hyperalgesia to pinprick assessed between 20 and $44 \mathrm{~h}$ after irradiation $(0$ to $24 \mathrm{~h}$ after drug administration). The ' -20 h' point expresses the expected value before the irradiation, that is the absence of secondary hyperalgesia. Data are expressed as means $\pm \mathrm{SD} ; \mathrm{N}=11$.

Hyperalgesia to mechanical stimuli was still present in both zones $44 \mathrm{~h}$ after UVB exposure $(\mathrm{MPT}=72.4 \pm 53.0 \mathrm{~g}$ and 134.6 $\pm 61.7 \mathrm{~g}$, respectively). Fig. 3 depicts the decrease of the MPT induced by UVB irradiation when compared to the control skin zone and its mean stability throughout the study day. Furthermore, the UVB irradiation also resulted in the development of an area of secondary hyperalgesia of $21.3 \pm$ $11.3 \mathrm{~cm}^{2}$, which was measured $20 \mathrm{~h}$ after the irradiation. The observed intra- and inter-individual variability for this parameter was $29.7 \%$ and $49.8 \%$, respectively (Table 1). However, two of the eleven volunteers did not develop hyperalgesia to pinprick outside the irradiated site during one of the four study sessions. The area of secondary hyperalgesia also remained remarkably stable throughout the day, with a coefficient of variation of $14.4 \%$ for within-subject within-day measures, as shown in Fig. 4. A significant shrinking from $21.3 \pm 11.3$ $\mathrm{cm}^{2}$ to $17.7 \pm 11.4 \mathrm{~cm}^{2}$ after $44 \mathrm{~h}(p<0.017)$ was observed.

\section{Discussion}

The present clinical study was mainly aimed at assessing the existence of an additive effect of a combination of paracetamol with ketorolac and exploring the putative mechanism of the interaction by conducting a PK/PD study in healthy human volunteers. For this purpose, tools were developed and validated for characterizing the PK and PD of the study drugs. The bioanalytical method that was developed for assessing the PK is the subject of another article. [12]

The PD was assessed using the sunburn model in a simplified form ${ }^{[18]}$ as an experimental model of pain. In the modified model, the MED was estimated on the basis of the volunteers' skin type in order to save time. From a methodological point of view, this clinical study was aimed at validating the simplified sunburn model in the research unit for the pharmacological profiling of a combination of analgesics and validating the model in terms of stabil- ity and variability. Sycha et $a l .{ }^{[18]}$ demonstrated an effect of ibuprofen on heat pain threshold and on heat pain tolerance with this modified model. At present, no single orally administered analgesic agent can completely and consistently relieve acute pain of moderate to severe intensity. Thus, the development of combination analgesics having distinct modes of action with the aim to provide additive effects with fewer side effects is an interesting approach. It has been found that the simplified UVB model is suitable for the assessment of the pharmacology of a combination of central and peripheral analgesics. This finding is important for further pharmacological studies addressing central and peripheral antihyperalgesic effects of new drug combinations. Moreover, no adverse events associated with the UVB irradiation were reported during the trial, which makes this model suitable for its use in healthy volunteers early in clinical drug development.

It was observed that this simplified sunburn model induced primary and secondary hyperalgesia, which showed consistent stability for a period between 20 and $26 \mathrm{~h}$ after the UVB irradiation. In addition, it was demonstrated that primary and secondary hyperalgesia were still present $44 \mathrm{~h}$ after the irradiation, but that the area of secondary hyperalgesia tended to decrease after this time interval. However, it should be mentioned that two volunteers did not develop an area of secondary hyperalgesia during one of the four study sessions. This might indicate that the simplified UVB model would not be as reproducible as when used according to individual MED. Further, very small intra-individual variability was observed for the HPT, with coefficients of variation of $1.5 \%$ and $2.6 \%$ for within- and between-day measurements, respectively. The UVB irradiation produced less variable hyperalgesia to heat stimuli than the burn injury, for which the coefficients of variation of $17 \%$ and $9 \%$ were obtained for within- and betweenday measurements, respectively. ${ }^{[21]}$ The variability was found to be larger for the MPT and the area of secondary hyperalgesia outcome variables; however, the intraindividual variability was always smaller than the inter-individual variability. This suggests that a crossover rather than parallel-group design should be employed to allow reasonable sample sizes for studies comparing treating effects. The betweensubject variability obtained in this study for MPT was comparable to the results obtained by Chassaing et al. ${ }^{[20]}$ after freezeinduced hyperalgesia. The variability observed for the UVB model was considerably smaller than that for the intradermal capsaicin model, in which the intra- and inter-individual variability was 140 and $787 \%$, respectively. ${ }^{[22]}$ 
In conclusion, this clinical study in healthy volunteers demonstrated that the simplified UVB model showed consistent within-day stability and between-day repeatability for primary hyperalgesia to heat stimuli. The variability to mechanical stimuli was higher; however, a crossover design would allow reasonable sample sizes. Secondary hyperalgesia to pinprick showed the same stability and variability when compared to MPT, but was however absent in two volunteers in one of four study sessions. Thus, it can be suggested that the simplified UVB model is suitable for pharmacological studies assessing only primary hyperalgesia; however, when assessing secondary hyperalgesia, the model based on individual MED determination might be even more reproducible.

\section{Acknowledgements}

This work has received financial support from the Department of anaesthesiology, pharmacology and intensive care of the University Hospitals of Geneva. This work has been awarded by the scientific board of the annual research meeting of the Department in June 2008.

Received: February 5, 2012

[1] M. Hyllested, S. Jones, J. L. Pedersen, H. Kehlet, Br. J. Anaesth. 2002, 88, 199.

[2] J. Romsing, S. Moiniche, J. B. Dahl, Br. J. Anaesth. 2002, 88, 215.

[3] C. K. Ong, R. A. Seymour, P. Lirk, A. F. Merry, Anesth. Analg. 2010, 110, 1170.

[4] A. Bertolini, A. Ferrari, A. Ottani, S. Guerzoni, R. Tacchi, S. Leone, CNS Drug Rev. 2006, 12, 250.

[5] D. M. Aronoff, J. A. Oates, O. Boutaud, Clin. Pharmacol. Ther. 2006, 79, 9 .

[6] G. Pickering, M. A. Loriot, F. Libert, A Eschalier, P. Beaune, C. Dubray, Clin. Pharmacol. Ther. 2006, 79, 371.

[7] G. Pickering, V. Esteve, M. A. Loriot, A Eschalier, C. Dubray, Clin. Pharmacol. Ther. 2008, 84, 47.

[8] C. Mallet, L. Daulhac, J. Bonnefont, C. Ledent, M. Etienne, E. Chapuy, F. Libert, A. Eschalier, Pain 2008, 139, 190.
[9] A. Ottani, S. Leone, M. Sandrini, A. Ferrari, A Bertolini, Eur. J. Pharmacol. 2006, 531, 280.

[10] J. N. Cashman, Drugs 1996, 52 Suppl 5, 13.

[11] M. Burian, G. Geisslinger, Pharmacol. Ther. 2005, 107, 139.

[12] K. R. Ing-Lorenzini, J. A. Desmeules, M. Besson, J. L. Veuthey, P. Dayer, Y. Daali, J Chromatogr A 2009, 1216, 3851 .

[13] K. Ing Lorenzini, M. Besson, Y. Daali, D Salomon, P. Dayer, J. Desmeules, Basic Clin. Pharmacol. Toxicol. 2011, 109, 357.

[14] L. Arendt-Nielsen, M. Curatolo, A. Drewes, Curr. Opin. Investig. Drugs 2007, 8, 41

[15] C. Staahl, A. E. Olesen, T. Andresen, L. ArendtNielsen, A. M. Drewes, Br. J. Clin. Pharmacol. 2009, 68, 322.

[16] B. Gustorff, S. Anzenhofer, T. Sycha, S. Lehr, H. G. Kress, Anesth. Analg. 2004, 98, 173

[17] C. Staahl, A. M. Drewes, Basic. Clin. Pharmacol. Toxicol. 2004, 95, 97.

[18] T. Sycha, B. Gustorff, S. Lehr, A. Tanew, H. G Eichler, L. Schmetterer, Br. J. Clin. Pharmacol. 2003, 56,165 .

[19] D. Yarnitsky, J. L. Ochoa, Pain 1990, 40, 85.

[20] C. Chassaing, J. Schmidt, A. Eschalier, J. M. Cardot, C. Dubray, Br. J. Clin. Pharmacol. 2006, 61, 389 .

[21] J. L. Pedersen, H. Kehlet, Pain 1998, 74, 139.

[22] A. Hughes, A. Macleod, J. Growcott, I. Thomas, Pain 2002, 99, 323. 